\title{
Outcomes in Thread Lift for Facial Rejuvenation: a Study Performed with Happy Lift ${ }^{\mathrm{TM}}$ Revitalizing
}

\author{
Antonella Savoia • Ciro Accardo • Fulvio Vannini • \\ Basso Di Pasquale $\cdot$ Alfonso Baldi
}

To view enhanced content go to www.dermtherapy-open.com

Received: December 5, 2013 / Published online: January 17, 2014

(C) The Author(s) 2014. This article is published with open access at Springerlink.com

\begin{abstract}
Introduction: Barbed suture lifting is a minimally invasive surgical technique for facial rejuvenation. This study examined the efficacy and associated risks with this procedure, using a new synthetic, monofilament suspension thread named "Happy Lift ${ }^{\mathrm{TM}}$ Revitalizing" (Promoitalia International S.r.l, Naples, Italy).
\end{abstract}

Methods: All the patients had average aging signs and required a lifting of modest degree. A total of 37 thread lifts were performed over a 24-month period.

Results: In the majority of patients (89\%), the results obtained were considered satisfactory. The incidence of complications was low. Only

Electronic supplementary material The online version of this article (doi:10.1007/s13555-014-0041-6) contains supplementary material, which is available to authorized users.

A. Savoia - C. Accardo · F. Vannini - B. Di Pasquale Promoitalia Group S.p.A., Pozzuoli, Naples, Italy

A. Baldi $(\varangle)$

Department of Environmental, Biological, Pharmaceutical Sciences and Technologies, Second University of Naples, Via L. Armanni 5, 80138 Naples, Italy

e-mail: alfonsobaldi@tiscali.it
$6 \%$ of the patients had slight post-operation asymmetry that was easily corrected. Minor complications experienced by patients included small ecchymosis (62\%), mild erythema (40\%), small hemorrhage (25\%), mild transitory esthesia (6\%) and mild post-operation tumefaction (40\%). Histopathological and ecographic analyses were performed on the treated skin of selected patients, demonstrating that the lifting effect is guaranteed and fortified by the cutaneous reaction that appears along the length of the thread.

Conclusion: Thread lift with "Happy $\mathrm{Lift}^{\mathrm{TM}}$ Revitalizing" is a safe procedure associated with minor complications, when performed on cohorts of patients requiring a facial lifting of modest degree.

Keywords: Barbed sutures; Dermatology; Facial ptosis; Facial rejuvenation; Happy $\mathrm{Lift}^{\mathrm{TM}}$ Revitalizing; Thread lift

\section{INTRODUCTION}

As we age, the scaffolding dermal facial skin, as well as the fat component, weakens. The affected areas generally include the cheeks, 
eyebrows, mandibular area and neck. This is attributable to the fact that the connective tissue of the skin becomes thinner and elastic fibers undergo a collapse [1]. This dermatocalasis of the facial and neck soft tissues, including the superficial muscular aponeurotic system (SMAS) and the muscular tissue, is the cause of the distinctive aging signs of the face [2]. Among other facial aging signs, we can highlight the profile of the mandibular margin, which is not clear anymore, displaying its downfall (down aging of the jaw line); the forehead has horizontal wrinkles at which other vertical ones are added to the glabellar area; the zygomatic malar region (middle face) displays a downward sliding; the lachrymal furrow appears and the naso-buccal and mandibular-bucco deepens; the skin of the eyelid becomes flabby and protrudes in correspondence to the lower eyelid due to production of adipose bubbles; and finally, plasmatic parcel and cutaneous flabbiness disappear from the neck [3].

The facelift to correct facial aging has been, so far, an elaborate and complicated procedure that takes a long time to recover from [4]. The recent introduction of absorbable barbed sutures producing a lifting action for this type of aging can be a good alternative to more invasive procedures [5-13]. The plugs present on the surface of the wires allow the combination with other non-surgical rejuvenation procedures, such as botulinum toxin, which has a 6-month temporary effect, or substances with a transient and volumizing filler effect. Other non-surgical procedures include radiofrequency that is limited to treating only very superficial wrinkles and mesotherapy, which deposits the so-called useful chemicals just under the skin, presumably increasing their usefulness in skin rejuvenation and improvement of the tone [14]. The spines along the course of the wire act as a hook to lift and support one area of the face whilst relaxed. The plugs open like an umbrella to form a support structure that lifts the sagging skin $[5,7]$. It is a soft approach that allows a safe reduction of the signs of aging. Depending on the material that makes up the suture and filling threads, we distinguish them as non-absorbable and absorbable threads [10].

In this study, we have analyzed a new type of re-absorbable barbed suture, "The Happy Lift ${ }^{\mathrm{TM}}$ Revitalizing" range. The aim of this study was to evaluate the surgical outcomes associated with this device in relation to eyebrow lifting, forehead lifting, mid-face lifting (zygomatic malar), upper-mandibular region lifting and high cervical region lifting.

\section{MATERIALS AND METHODS}

\section{Barbed Suture}

The "Happy Lift ${ }^{\mathrm{TM}}$ Revitalizing range" is an absorbable, monofilament, suspension thread of synthetic origin (caprolactone) manufactured by Assut Europe Spa for Promoitalia International spa (Promoitalia International S.r.l, Naples, Italy). This product belongs to the category 07 of inactive implanted devices, in the re-absorbable thread group, and in class III of medical devices [15]. Moreover, the device has the CE mark 0373 released by the Istituto Superiore della Sanità dell'Italia (Italian Board of Health, Rome, Italy).

This study has utilized only lifting threads. In particular, Free floating for zygomatic and mandibular area, Double needle for eyebrow and neck area and Ancorage for mid-face lifting (zygomatic malar) in patients over 60 years. In detail, Double needle threads are convergent bidirectional threads (length 12-23 cm, 2.0 usp caliber) with straight needle (lengths 9 or 
$15 \mathrm{~cm}, 24 \mathrm{G}$ caliber) at the ends. Free floating threads are convergent bidirectional threads (length $12-23 \mathrm{~cm}, 2.0$ usp caliber) which are introduced into the hypoderm with the assistance of cannula (Length 9/15 cm, 21G caliber) and Ancorage are unidirectional, barbed threads (Length $12.5 \mathrm{~cm}, 2.0$ usp caliber).

\section{Patients}

Thirty-seven female patients, aged between 37 and 65 years old, participated in the study between December 2010 and December 2012. All procedures were performed in anti-aging centers of Promoitalia (Promoitalia International S.r.l, Naples, Italy). All patients had average aging signs and required a lifting of modest degree. The following cases have been regarded suitable for lifting operations: marked naso-labial fold; slightly defined mandibular contour; general aspect of an aged face (eye wrinkles, forehead wrinkles) and relaxed neck and chin with skin-folds. The exclusion criteria in the study were patients in whom aging signs, such as facial lipoatrophy, evident laxity and cutaneous hypotonicity, were more advanced and evident and patients with an excessive overabundance of skin, thus requiring a removal. Patients who requested immediate results, excessive lifting, traditional lifting, and those with very advanced cutaneous-muscular prolapse, pathologies to diagnose or with diagnosed pathologies were also excluded. Finally, for patients over 45 , the recourse to this method was considered valid only when linked to ancillary techniques such as Phenol or Trichloroacetic Acid (TCA) peeling, or laser therapies $\left(\mathrm{CO}_{2}\right.$, Radiofrequency), or autologous fat transplant (in this case, it was necessary to restore the sub-dermic volume). Based on the different treatments, we divided the 37 patients into three groups. Group 1 contained five patients treated with Ancorage for mid-face lifting. Group 2 contained eight patients treated with Free floating for zygomatic and mandibular area and Group 3 consisted of twenty-four patients treated with Double needle for eyebrow and neck area. No patient modified his/her lifestyle and consent was given to the protocol proposed. All patients underwent a preventative checkup, including a complete anamnesis, and had ultrasound and photographic evaluation.

\section{Compliance with Ethics}

All procedures followed were in accordance with the ethical standards of the responsible committee on human experimentation (institutional and national) and with the Helsinki Declaration of 1975, as revised in 2000 and 2008. Informed consent was obtained from all patients for being included in the study.

\section{Treatment Protocol}

The "Happy Lift $^{\mathrm{TM}}$ Revitalizing range" was inserted in hypodermic tissues of the face, upper the SMAS, and in subcutaneous tissues of the neck and forehead. Local anesthesia was performed with 1-2\% Lidocaine, diluted Epinephrine $(1 / 200,000 \mathrm{cc})$ and the addition of diluted sodium hydrogen carbonate $\left(\mathrm{NaHCO}_{3}\right)$ (1/9 cc).

The Free Floating threads were introduced in flabby tissues of zygomatic area with a cannula needle $(20 / 21 \mathrm{G}$ caliber $\times 6 \times 1,2 \mathrm{~mm} \times 15$, $24 \mathrm{~cm}$ ) and subsequently, the cannula was removed, leaving the thread in its place. The thread, with bidirectional and convergent barbs towards the center, was inserted inside the needle carefully so that the barbs that had to maintain their efficiency were not damaged. The mechanical action exercised by the two 
halves of the thread had to be symmetrical and of the same entity; otherwise, the sliding of the thread in the insertion level and the "exiting" from one of the access holes was possible. The time necessary for the complete treatment required was an average of 35-55 $\mathrm{min}$.

The Double needle threads were utilized for the treatment of the forehead, mandibular area and the neck. The protocol used for the revival of the eyebrow suggested the insertion of both straight needles at a point located in correspondence to the hairline. One proceeds vertically downward until reaching the eyebrow where both needles protrude. The course must be tangential to the bone surface and within the subcutaneous tissue. The median needle reenters in the same exit hole and traces the eyebrow to exit the nasal edge. The needle placed more laterally undergoes the same path, symmetrically (Fig. 1a). The protocol used for the redefinition of the mandibular contour involves the use of a wire introduced through a straight needle in correspondence to the mandibular angle through two adjacent inlet holes. The first needle goes along the ramus of the lower jaw to the temporal region and the second runs on the horizontal ramus of the mandible for $4-5 \mathrm{~cm}$ (Fig. 1b). Finally, for the treatment of the neck, a wire double needle is introduced in correspondence to the aponeurosis of platysma with both needles, but in an alternating manner. It proceeds to the region of the sternum cleido mastoid drawing a "V" open towards the outside (Fig. 1c).

The time necessary for a complete treatment was, on average $20-40 \mathrm{~min}$. The threads may displace themselves from their primal insertion position in the first 10 days following traumas and therefore, loose the "gathering" established with the neighboring tissues. Therefore, during the first 3 weeks after the operation, patients were not permitted to take part in any kind of intense physical activity, to exercise any violent or direct pressure on the areas in which the threads were inserted or to perform vigorous rubbing on the facial skin and neck.

\section{Evaluation Protocol}

Photographs of the treated areas taken from different angles and with varying visualizations (using the same positions of the subject, light and the exposure of the shot) were taken after 25 days and at 2, 6 and 12 months after the operation. Two facial shots have been used to standardize the images and to try and obtain useful iconographic documentation.

For the ultrasonic study of the skin and subcutaneous tissue, Mindray ${ }^{\mathrm{TM}}$ DP 9900 Plus (Mindray Medical International, Milan, Italy), indicated for the bidirectional observation of the connective tissue, was used to verify the fibrosis induced by Happy Lift ${ }^{\mathrm{TM}}$ in fatty tissues of the face and neck.
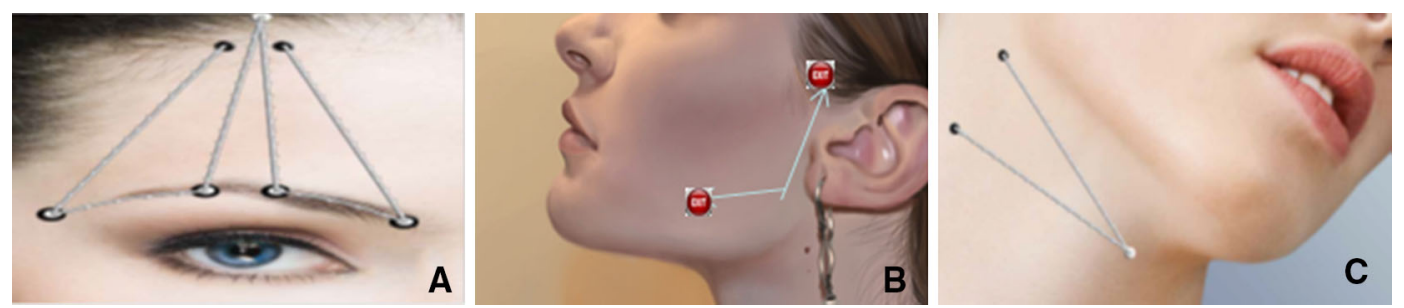

Fig. 1 a Double needle for the treatment of the eyebrow; b double needle for the redefinition of the mandibular contour; c double needle for the treatment of the neck 
Table 1 Global Aesthetic Improvement Scale (GAIS)

\begin{tabular}{lll}
\hline & Degree & Description \\
\hline 1 & Exceptional improvement & Excellent corrective result \\
2 & Very improved patient & Marked improvement of the appearance, but not completely optimal \\
3 & Improved patient & $\begin{array}{c}\text { Improvement of the appearance, better compared with the initial } \\
\text { condition, but a touch-up is advised }\end{array}$ \\
4 & Unaltered patient & $\begin{array}{c}\text { The appearance substantially remains the same compared with the } \\
\text { original condition }\end{array}$ \\
5 & Worsened patient & The appearance has worsened compared with the original condition \\
\hline
\end{tabular}

Histopathological analysis was performed on two patients treated with Free Floating in the zygomatic area. Bioptic drawings were carried out on the left and right cheeks 60 days after the operation, when a reduction in the entity of the oscillating ptosis ranging between 100\% and $75 \%$ was obtained. Excised tissue samples were transferred in $4 \%(\mathrm{~m} / \mathrm{v})$ paraformaldehyde solution and embedded in paraffin. Sections of $5 \mu \mathrm{m}$ were stained with hematoxylin-eosin, hematoxylin-van Gieson, and periodic acidSchiff (PAS)-Alcian blue hematoxylin.

Ultrasound evaluation was carried out 90 days after the treatment to identify the structural modifications to which the skin had been subjected to, using B-mode ultrasonography.

The results were defined with a score derived from the Global Aesthetic Improvement Scale (GAIS), used as a reference parameter (Table 1). The values in the scale were determined independently by Fulvio Vannini and Antonella Savoia and their concordance was $100 \%$. A summary of the patients' characteristics and the GAIS scores obtained are provided in Table 2 .

\section{Statistical Analysis}

Descriptive analysis was made using median values and 95\% Confidence Interval (CI). The differences in the GAIS scores in the different time-points of each group were performed using the Wilcoxon's test for nonparametric dependent continuous variables. SPSS software (version 17.00, SPSS, Chicago, USA) was used for statistical analysis. A $P$ (two tailed) value of $<0.05$ was considered to indicate statistical significance.

\section{RESULTS}

In 33 patients (89\%), the result was considered satisfactory, including 24 patients (65\%) with excellent results and 9 patients (24\%) with a good result. The result was considered unsatisfying for the final four patients (11\%). This optimal cosmetic effect was statistically significant not only immediately after the treatment, but also after 6 months $(P=0.005)$. Indeed, there was not a statistically significant difference between the two evaluations.

None of the patients asked for the removal of the threads immediately after the operation and none of them encountered important complications.

During the first 2 weeks, two patients from Group 1 had slight post-operation asymmetry. This was immediately corrected by slightly compressing the surgeons' right hand thumb in the omo-lateral region of the hyper-corrected 
Table 2 Clinical characteristics of the patients, different treatments and Global Aesthetic Improvement Scale (GAIS) evaluation

\begin{tabular}{|c|c|c|c|c|c|}
\hline Patient no. & Age (years) & Areas treated & Threads type & $\begin{array}{l}\text { GAIS1 after } \\
\text { the treatment }\end{array}$ & $\begin{array}{l}\text { GAIS2 after } \\
6 \text { months }\end{array}$ \\
\hline 1 & 52 & Zygomatic area & Free floating & 2 & 1 \\
\hline 2 & 54 & Neck & Double needle & 3 & 2 \\
\hline 3 & 63 & Mid-face & Ancorage & 1 & 1 \\
\hline 4 & 41 & Mandibular contour & Double needle & 1 & 1 \\
\hline 5 & 42 & Mandibular contour & Double needle & 1 & 1 \\
\hline 6 & 64 & Mid-face & Ancorage & 2 & 1 \\
\hline 7 & 59 & Zygomatic area & Free floating & 1 & 1 \\
\hline 8 & 52 & Neck & Double needle & 3 & 1 \\
\hline 9 & 58 & Zygomatic area & Free floating & 2 & 2 \\
\hline 10 & 48 & Eyebrow & Double needle & 2 & 1 \\
\hline 11 & 50 & Neck & Double needle & 3 & 2 \\
\hline 12 & 57 & Zygomatic area & Free floating & 2 & 2 \\
\hline 13 & 49 & Eyebrow & Double needle & 3 & 2 \\
\hline 14 & 50 & Eyebrow & Double needle & 2 & 2 \\
\hline 15 & 50 & Neck & Double needle & 1 & 1 \\
\hline 16 & 45 & Eyebrow & Double needle & 2 & 1 \\
\hline 17 & 38 & Mandibular contour & Double needle & 1 & 1 \\
\hline 18 & 60 & Mid-face & Ancorage & 2 & 2 \\
\hline 19 & 61 & Mid-face & Ancorage & 3 & 2 \\
\hline 20 & 54 & Zygomatic area & Free floating & 1 & 1 \\
\hline 21 & 50 & Mandibular contour & Double needle & 2 & 1 \\
\hline 22 & 51 & Neck & Double needle & 3 & 2 \\
\hline 23 & 44 & Eyebrow & Double needle & 3 & 2 \\
\hline 24 & 48 & Mandibular contour & Double needle & 1 & 1 \\
\hline 25 & 62 & Mid-face & Ancorage & 4 & 2 \\
\hline 26 & 47 & Mandibular contour & Double needle & 1 & 1 \\
\hline 27 & 48 & Zygomatic area & Free floating & 3 & 1 \\
\hline 28 & 41 & Mandibular contour & Double needle & 2 & 1 \\
\hline 29 & 51 & Eyebrow & Double needle & 2 & 2 \\
\hline 30 & 54 & Neck & Double needle & 4 & 2 \\
\hline 31 & 48 & Eyebrow & Double needle & 4 & 3 \\
\hline
\end{tabular}


Table 2 continued

\begin{tabular}{|c|c|c|c|c|c|}
\hline Patient no. & Age (years) & Areas treated & Threads type & $\begin{array}{l}\text { GAIS1 after } \\
\text { the treatment }\end{array}$ & $\begin{array}{l}\text { GAIS2 after } \\
6 \text { months }\end{array}$ \\
\hline 32 & 54 & Mandibular contour & Double needle & 1 & 1 \\
\hline 33 & 43 & Eyebrow & Double needle & 2 & 2 \\
\hline 34 & 48 & Mandibular contour & Double needle & 1 & 1 \\
\hline 35 & 52 & Zygomatic area & Free floating & 3 & 2 \\
\hline 36 & 47 & Eyebrow & Double needle & 4 & 2 \\
\hline 37 & 49 & Zygomatic area & Free floating & 2 & 1 \\
\hline
\end{tabular}

face. The result after this exercise achieved the patients' complete satisfaction. In two patients from Group 2 and three patients of Group 3, a more severe asymmetry was corrected by introducing one or two more threads in the area of the hyper-corrected face. This operation was performed about 30-45 days after the first operation.

The most frequent minor complication was the presence of small ecchymosis in 23 patients (62\%). The great majority of this ecchymosis concerned the temporal area for the patients of Group 1 and 2. Mild erythema was noticed in 15 patients $(41 \%)$. These clinical signs were protracted for a maximum of 3 weeks and did not request any treatment. In nine patients (24\%), a small hemorrhage appeared immediately after the introduction of the thread in the sub-dermal layer of the skin. It ceased either by mechanical compression or in the moment during which the extremities were cut off. Mild transitory esthesia of a maximum of 2 months was encountered in two patients (5\%). Mild post-operation tumefaction was observed in 15 patients (41\%). These tumefactions were successfully treated with non-steroidal drugs. Table 3 summarizes all the complications encountered and the different treatments used. A patient treated for the
Table 3 Complications encountered and treatments used

\begin{tabular}{lll}
\hline $\begin{array}{l}\text { Patients } \\
\text { group no. }\end{array}$ & Complication & Solution \\
\hline 1 & Asymmetry & Slight compression \\
$1-2$ & Asymmetry & $\begin{array}{c}\text { Introduce one or more } \\
\text { threads }\end{array}$ \\
$1-2-3$ & Ecchymosis & N/A \\
$1-2-3$ & Erythema & N/A \\
$1-2-3$ & Small & Mechanical \\
& hemorrhage & compression \\
$1-2$ & Tumefaction & Non-steroidal drugs \\
\hline
\end{tabular}

redefinition of the mandibular contour is depicted in Fig. 2.

The thread was not visible because the insertion area was sub-dermal and the thread was transparent. All the blood, urine and electrocardiogram tests were normal during the testing period. The operation had minimal or no side effects. All the participants were able to return to their everyday activities 3 days after the treatment.

\section{Histopathological Analysis}

The histological analysis was performed 2 and 6 months after the thread lift on two different patients. In the first patient, 2 months after the 

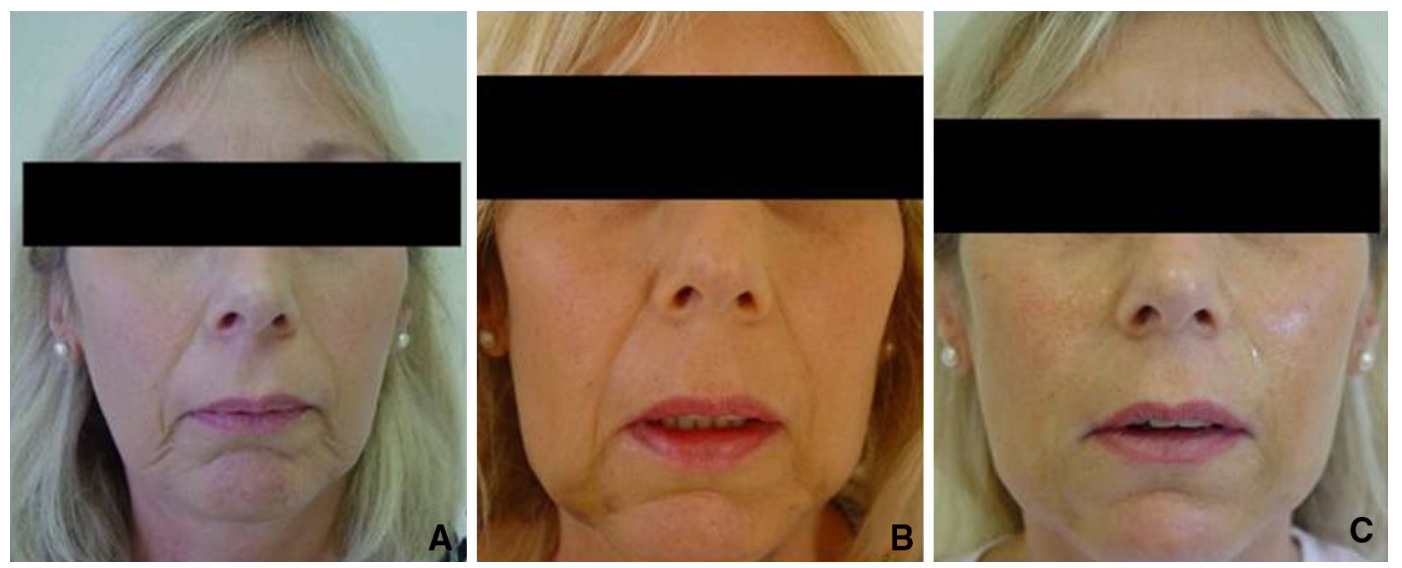

Fig. 2 Exemplificative case of facial rejuvenation. A patient treated for the redefinition of the mandibular contour. a Before the treatment. b Immediately after the treatment. c 6 months after the treatment

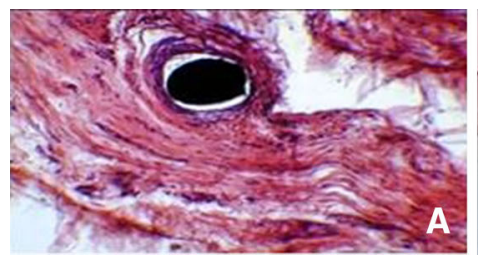

Fig. 3 a Fragment of skin removed 2 months after the procedure from the right cheek containing a white microscopic filament (Happy Lift ${ }^{\mathrm{TM}}$ Revitalizing). ( $\mathrm{H} \& \mathrm{E}$, original magnification $\times 20$ ). b Fragment of skin taken from the right cheek 60 days after the operation. The dermis next to the areas of the right zygomatic malar, where the "Happy Lift ${ }^{\mathrm{TM}}$ Revitalizing" thread is inserted, normally represents annexation structures and vessels with

procedure, observation with an optical microscope enabled the detection of a fragment of thread in the subcutaneous tissue to which a connective structure adhered. The latter was structured around a roughly ovoidshaped cavity with a maximum diameter of around $0.3 \mathrm{~mm}$ (Fig. 3a). The internal section of this structure, looking into the cavity, was made up of larger and rounder "fibroblastic" cells surrounding a "Free floating" thread that appears dark following the histological preparations, but when fresh, was white. On the other hand, on a fragment of skin taken from the left cheek 60 days after the operation, no signs of inflammation; an increase in the thickness of the papilla can also be noticed. ( $\mathrm{H} \& \mathrm{E}$, original magnification $\times 10$ ). c Fragment of subcutaneous tissue removed 2 months after the procedure, showing a connective structure forming a ring with a thickness of around $70 \mu \mathrm{m}$ around a cavity seat of the "Happy Lift ${ }^{\mathrm{TM}}$ Revitalizing". $(\mathrm{H} \& \mathrm{E}$, original magnification $\times 20)$

derma and epidermis with a normal aspect and with a minuscule part of subcutaneous lacking abnormal structures were seen. In this derma adjacent to the insertion areas in the left uppermandible region of the Happy $\mathrm{Lift}^{\mathrm{TM}}$ Revitalizing thread vessels and annexation structures are normally represented and there were no signs of inflammation (Fig. 3b). Interestingly, in the biopsy performed after 2 months on the second patient, it is possible to detect some connective structures in the subcutaneous tissue, which form a ring with a thickness of around $70 \mu \mathrm{m}$ around a cavity seat of the "Happy Lift $^{\mathrm{TM}}$ Revitalizing". The 


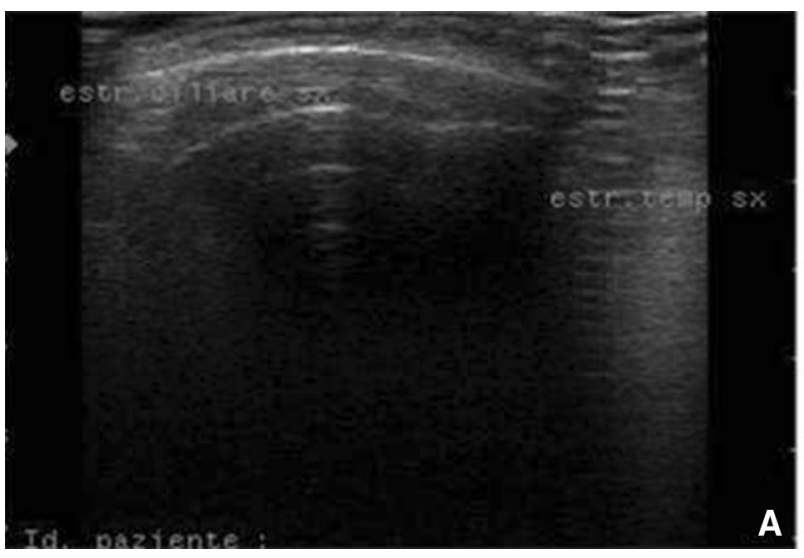

Fig. 4 Ultrasound evaluation of the treated zygomatic area of two different patients 90 days from the date of the implant. a The hyper-echogenicity due to the marginal

innermost part is made up of lager and rounder fibroblastic cells crammed into $2-4$ chains with a higher number of dilated vessels. There were also rare giant multinucleate cells with histiocytic features (histiocytes) present. The peripheral part of the connective structure appeared to be made up of less young connective tissue with a poor number of dilated fibrohistiocytic and connective cells, thickened with a small amount of vessels (Fig. 3c). To note, we detected no signs of acute inflammation, necrosis or other significant pathological phenomenon in all the specimens analyzed.

\section{Ecotomographic Evaluations in B mode}

This analysis confirms, through the hyperechogenicity due to the marginal fibrosis around the threads, the persistence evidence of the Happy Lift thread after 90 days from the implant in zygomatic area. Indeed, fibrosis promotes a natural repositioning of the tissues and stimulates collagen regeneration with tissue toning. The fibrotic process made is highlighted as a hyper echoic area at two-dimensional ultrasonography (Fig. 4a).

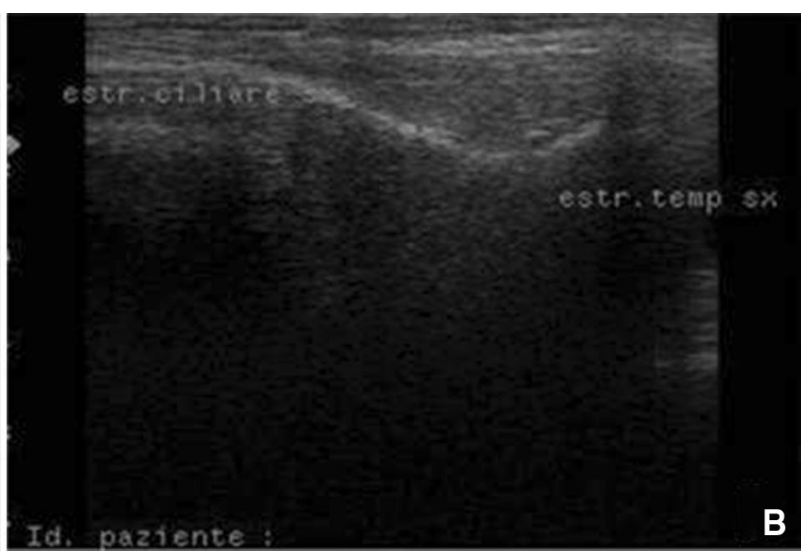

long-lasting fibrosis around the threads is evident; b The particular stereotaxic disposition of the threads route is to be noticed

Moreover, it confirms that the specific spatial geometry of the surface of the barbed thread is responsible for the marginal fibrosis highlighted in the ecographic image in B mode. In fact, it is possible to observe in Fig. $4 \mathrm{~b}$, the particular stereotaxic disposition of the threads route that placed according to the standard map, is arranged like a spoon course that embraces the lowest point of the down aging of the jaw line.

\section{DISCUSSION}

Innovations in operative techniques generally contribute to enhanced results, greater patient happiness, and a decrease in operative morbidity $[1,4]$. The results of this study have shown that the use of the "Happy $\mathrm{Lift}^{\mathrm{TM}}$ Revitalizing" is a safe and successful technique for facial rejuvenation. The immediate effect is the lifting of the tissue, due to the mechanical action produced by the thread, which contrasts the falling of the area treated. This is possible due to the arrangement of the threads' barbs, disposed in two directions (divergent and opposite), compared to the middle point of the thread. The reaction strength produced on both its sides, in fact, allows the connection of 
the tissue on each barb that functions as a hook and at the same time, prevents the sliding and movement of the thread in the two opposite directions. Thanks to this specific geometry of the "Happy Lift ${ }^{\mathrm{TM}}$ Revitalizing", anchorage points are not required on the superior and inferior part of the facial anatomic region to lift. Once positioned in the subcutaneous tissue, they will continue to practice their sustaining action on the tissues. It is an advantage compared to the unidirectional barbed threads as these barbs are positioned in one direction. Therefore, even if widespread, they lift the tissue in only one direction $[10,13,14]$. The histological valuations have highlighted that the sub-dermal suspension thread used in this study connects itself to the high and lateral dermis retinacula cutis system, to the SMAS and to the downward muscular bands to exercise an effective anchoring and lifting action. In detail, the histological modifications induced by the thread "Happy Lift $^{\mathrm{TM}}$ Revitalizing" can be summarized in the following points. A homogeneous fibrous capsule forms around the thread that continues to assure the tissues' traction and compactness. Fibrosis presents an intensive new vascular genetic activity in its context, with dilated vessels. The dermis papillae appear to have a greater thickness indicating the growth of the collagen interstitial component. Finally, a significant acute inflammatory response is not elicited by the treatment. Therefore, it is possible to claim that the lifting effect is guaranteed and fortified by the cutaneous reaction (fibrosis) that appears along the length of the thread, and that remains effective and steady even when the thread is completely re-absorbed (after about a year). The re-absorption occurs due to the action of the histiocitary reticule system, which concretises a selective hydrolysis action of the re-absorbable thread from the outskirts towards the center.
This is also confirmed by the data produced with the ecographic analysis. It has to be emphasized that the GAIS evaluation has not been registered until 6 months after the procedure. Therefore, it could be possible that with a longer period of follow-up, some other side effects could appear.

The most important limits of this technique are that it is indicated for moderate cutaneous falling. For overabundant tissue, the prescription remains to be traditional lifting. Nevertheless, it must be underlined that the results encountered with different barber suture lifting have shown a significant number of adverse events and early recurrence [16, 17]. Therefore, the results of this study suggest that increasing the number of patients treated with the "Happy Lift ${ }^{\mathrm{TM}}$ Revitalizing" would confirm the good results obtained in this study. Finally, an important factor to be considered when analyzing the results obtained with this technique is the correct selection of the patients to be treated. For these medical devices, the most suitable candidates are those with mild aging signs and that necessitate a lifting of modest degree. In case of more advanced and evident signs of aging, patients must opt for traditional surgical options that are more invasive and direct. Therefore, severe selection criteria must be adopted when selecting the patients to be treated with this technique.

\section{CONCLUSION}

From our work, it appears that the sub-dermal suspension with the re-absorbable thread "Happy Lift ${ }^{\mathrm{TM}}$ Revitalizing" constitutes an efficient and safe procedure of mid-face lifting and rejuvenation of the superior cervical region of the face and neck. It is also possible to associate other methods that allow the 
optimizing of the complete facial rejuvenating effect with botulin, fillers, chemical peelers, photo-rejuvenation with a pulsed light, lipfilling, etc. [1, 3]. The operation with such threads does not require general anesthesia. It is virtually without bleeding or pain, intra- and post-operational and does not produce scars that are visible on the skin nor does it require post-operational recovery. The technique is practicable in day surgery and the patient may immediately return to everyday activities shortly following the procedure [14].

\section{ACKNOWLEDGMENTS}

Dr. A. Baldi is the guarantor for this article, and takes responsibility for the integrity of the work as a whole. No funding or sponsorship was received for this study or publication of this article.

Conflict of interest. Alfonso Baldi is scientific advisor of Promoitalia Group SpA.

A. Saviola, C. Accardo, F. Vannini and B. Di Pasquale are employees of Promoitalia International S.r.l, Naples, Italy.

Compliance with ethics guidelines. All procedures followed were in accordance with the ethical standards of the responsible committee on human experimentation (institutional and national) and with the Helsinki Declaration of 1975 , as revised in 2000 and 2008. Informed consent was obtained from all patients for being included in the study.

Open Access. This article is distributed under the terms of the Creative Commons Attribution Noncommercial License which permits any noncommercial use, distribution, and reproduction in any medium, provided the original author(s) and the source are credited.

\section{REFERENCES}

1. Helfrich YR, Sachs DL, Voorhees JJ. Overview of skin aging and photoaging. Dermatol Nurs. 2008;20:177-83.

2. Mack WP. Complications in periocular rejuvenation. Facial Plast Surg Clin North Am. 2010;18:435-56.

3. Nkengne A, Bertin C. Aging and facial changesdocumenting clinical signs, part 1: clinical changes of the aging face. Skinmed. 2012;10:284-9.

4. Chaffoo RA. Complications in facelift surgery: avoidance and management. Facial Plast Surg Clin North Am. 2013;21:551-8.

5. Wu WTL. Barbed sutures in facial rejuvenation. Aesthet Surg J. 2004;24:582-7.

6. Lycka B, Bazan C, Poletti E, Treen B. The emerging technique of the antiptosis subdermal suspension thread. Dermatol Surg. 2004;30:41-4.

7. Lee S, Isse N. Barbed polypropylene sutures for midface elevation. Arch Facial Plast Surg. 2005;7:55-61.

8. Isse NG, Fodor $\mathrm{PB}$. Elevating the midface with barbed polypropylene sutures. Aesthet Surg J. 2005;25:301-3.

9. Ruff G. Technique and uses for absorbable barbed sutures. Aesthet Surg J. 2006;26:620-8.

10. De Lorenzi CL. Barbed sutures, rationale and techniques. Aesthet Surg J. 2006;26:223-9.

11. Sulamanidze MA, Fournier PF, Paikidze TG, Sulamanidze GM. Removal of facial soft tissue ptosis with special threads. Dermatol Surg. 2002;28:367-71.

12. Sulamanidze MA, Paikidze TG, Sulamanidze GM, Neigel JM. Facial lifting with "APTOS" threads: featherlift. Otolaryngol Clin North Am. 2005;38:1109-17.

13. Horne DF, Kaminer MS. Reduction of face and neck laxity with anchored, barbed polypropylene sutures (Contour Threads). Skin Therapy Lett. 2006;11:5-7.

14. Villa MT, White LE, Alam M, Yoo SS, Walton RL. Barbed sutures: a review of the literature. Plast Reconstr Surg. 2008;121:102e-8e. 
15. Council Directive 93/42/EEC of the European Parliament and of the Council of 14 June 1993. http://www.ce-mark.com/MDD.pdf. Accessed 25 Nov 2013.

16. Garvey PB, Ricciardelli EJ, Gampper T. Outcomes in threadlift for facial rejuvenation. Ann Plast Surg. 2009;62:482-5.
17. Rachel JD, Lack EB, Larson B. Incidence of complications and early recurrence in 29 patients after facial rejuvenation with barbed suture lifting. Dermatol Surg. 2010;36:348-54. 